\title{
Material dependence of Casimir forces: gradient expansion beyond proximity
}

\author{
Giuseppe Bimonte, ${ }^{1,2}$ Thorsten Emig, ${ }^{3}$ and Mehran Kardar ${ }^{4}$ \\ 1) Dipartimento di Scienze Fisiche, Università di Napoli Federico II, Complesso Universitario MSA, Via Cintia, \\ I-80126 Napoli, Italy \\ ${ }^{2)}$ INFN Sezione di Napoli, I-80126 Napoli, Italy \\ ${ }^{3)}$ LPTMS, CNRS UMR 8626, Bât. 100, Université Paris-Sud, 91405 Orsay cedex, \\ France \\ ${ }^{4)}$ Massachusetts Institute of Technology, Department of Physics, Cambridge, Massachusetts 02139, \\ $U S A$
}

(Dated: 31 October 2018)

A widely used method for estimating Casimir interactions [H. B. G. Casimir, Proc. K. Ned. Akad. Wet. 51, 793 (1948)] between gently curved material surfaces at short distances is the proximity force approximation (PFA). While this approximation is asymptotically exact at vanishing separations, quantifying corrections to PFA has been notoriously difficult. Here we use a derivative expansion to compute the leading curvature correction to PFA for metals (gold) at room temperature. We derive an explicit expression for the amplitude $\hat{\theta}_{1}$ of the PFA correction to the force gradient for axially symmetric surfaces. In the non-retarded limit, the corrections to the Casimir free energy are found to scale logarithmically with distance. For gold, $\hat{\theta}_{1}$ has an unusually large temperature dependence.

The continual drive towards miniaturization of devices inevitably leads to scales were quantum effects are significant. In the realm of micromechanical systems (MEMS) - nano fabricated devices actuated by electrical bias- Casimir ${ }^{1}$ and van der Waals forces ${ }^{2}$ are paramount. These forces originate in quantum fluctuations of electromagnetic fields, and may cause devices to fail due to stiction. ${ }^{3}$ Due to their non-trivial dependence on shape and material properties, Casimir forces are difficult to compute. $^{4,5}$ A common method for estimating forces in a general setup is the proximity force approximation (PFA): First the force is calculated between two parallel plates at separation $d$ with the Lifshitz formula (as a function of dielectric response of the adjoining slabs $;^{6}$ ) then the geometry is taken into account by averaging over (appropriately defined) separations $d$ between adjoining surfaces. ${ }^{7}$ While this approximation is qualitatively wrong in special circumstances, ${ }^{8}$ it remains a useful tool in high precision experiments between surfaces of large radii of curvature $R$. In these cases PFA is asymptotically exact at small separations (for $d \ll R$ ). However, improvements in sensitivity of measurements warrant quantifying corrections to PFA which we undertake in this paper.

We consider a geometry consisting of two infinitely thick plates composed of homogeneous and isotropic dielectric materials, with permittivities $\epsilon_{1}(\omega)$ and $\epsilon_{2}(\omega)$. For simplicity we assume that one of the plates is a planeparallel slab, while the other is gently curved, and characterized by a smooth height profile $z=H(\mathbf{x})$, where $z$ is the local distance from the planar surface $\Sigma_{2}$, and $\mathbf{x}=\left(x_{1}, x_{2}\right)$ is the vector spanning $\Sigma_{2}$ (see Fig. 1). ${ }^{9}$ Following Refs. 10 and 11 we postulate that the Casimir free energy $\mathcal{F}[H]$ admits a local expansion of the form

$$
\mathcal{F}[H]=\mathcal{F}_{\mathrm{PFA}}[H]+\int_{\Sigma_{2}} d \mathbf{x} \alpha(H) \nabla H \cdot \nabla H+\rho^{(2)}[H]
$$

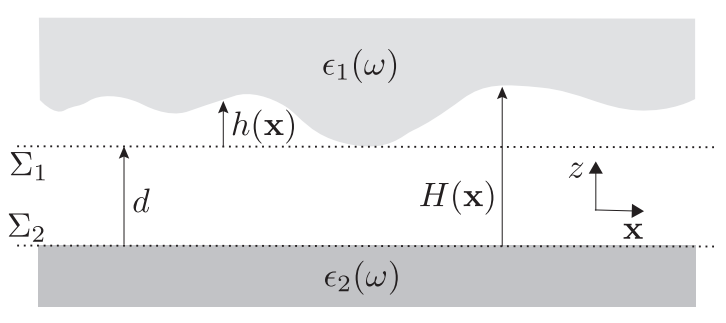

FIG. 1. Parametrization of a the profile of a gently curved dielectric surface near a flat dielectric plate.

where $\mathcal{F}_{\mathrm{PFA}}[H]$ represents the PFA free energy

$$
\mathcal{F}_{\mathrm{PFA}}[H]=\int_{\Sigma_{2}} d \mathbf{x} \mathcal{F}_{p p}(H)
$$

with $\mathcal{F}_{p p}(z)$ the free energy per unit area for two planeparallel dielectric plates of permittivities $\epsilon_{1}(\omega)$ and $\epsilon_{2}(\omega)$ at distance $z$, as given by the Lifshitz formula, ${ }^{6}$ and $\alpha(H)$ is a function to be determined. The quantity $\rho^{(2)}[H]$ in Eq. (1) represents corrections that become negligible if the curvature of the surface is small compared to the minimal distance $d$ between the surfaces. ${ }^{12}$

The function $\alpha(H)$ in Eq. (1) can be determined from a perturbative expansion of the Casimir free energy in the deformation profile $h(\mathbf{x})$, defined above the point of closest proximity as $H(\mathbf{x})=d+h(\mathbf{x})$, see Fig. 1. Note that the latter perturbation requires a small deformation amplitude while the gradient expansion relies upon small changes in the slope. However, it can be shown that under certain conditions (existence of perturbation theory in $h(\mathbf{x})$ and regularity of the involved kernels in momentum space) the gradient expansion of Eq. (1) follows from resumming the perturbative series for small in-plane momenta. If so, the expansion of the free energy in Eq. (1) 
has to match the perturbative expansion for $|h(\mathbf{x})| / d \ll 1$ at small momenta. The latter is given by

$$
\begin{aligned}
\mathcal{F}[d+h(\mathbf{x})] & =A \mathcal{F}_{p p}(d)+\mu(d) \tilde{h}(\mathbf{0}) \\
& +\int \frac{d^{2} \mathbf{k}}{(2 \pi)^{2}} \tilde{G}(k ; d)|\tilde{h}(\mathbf{k})|^{2}+\bar{\rho}^{(2)}[h],
\end{aligned}
$$

where $A$ is the surface area, $\mathbf{k}$ is the in-plane wave-vector, $\tilde{h}(\mathbf{k})$ is the Fourier transform of $h(\mathbf{x})$, and $\bar{\rho}^{(2)}[h]$ refers to higher order corrections. The function $\alpha(H)$ can now be determined if the kernel $\tilde{G}(k ; d)$ can be expanded to second order in $k$ (which as we shall see is the case for our problem). Indeed, matching the expansion

$$
\tilde{G}(k ; d)=\gamma(d)+\delta(d) k^{2}+o\left(k^{2}\right),
$$

to Eq. (1) leads to

$$
\mathcal{F}_{p p}^{\prime}(d)=\mu(d), \quad \mathcal{F}_{p p}^{\prime \prime}(d)=2 \gamma(d), \quad \alpha(d)=\delta(d),
$$

where a prime denotes a derivative with respect to $d$. For further justification of the gradient expansion we refer to the discussion after Eq. (12).

We now give an outline of the computation of the kernel $\tilde{G}(k ; d)$. The staring point is the scattering formula for the Casimir free energy $\mathcal{F},{ }^{13}$

$$
\mathcal{F}=k_{B} T \sum_{n \geq 0}^{\prime} \operatorname{Tr} \log \left[1-\mathbb{T}^{(1)} \mathbb{U} \mathbb{T}^{(2)} \mathbb{U}\right],
$$

where $k_{B}$ is Boltzmann's constant, $T$ the temperature, and the primed sum runs over Matsubara frequencies $\xi_{n}=2 \pi n k_{B} T / \hbar$ with the $n=0$ term weighted by $1 / 2$. In Eq. (6), $\mathbb{T}^{(j)}$ denotes the T-operator of plate $j$, evaluated for imaginary frequency $i \xi_{n}$. In a plane-wave basis $|\mathbf{k}, Q\rangle,{ }^{14}$ where $Q=E, M$ is the polarization, $E$ and $M$ denote respectively electric (transverse magnetic) and magnetic (transverse electric) modes. The translation operator $\mathbb{U}$ in Eq. (6) is diagonal with matrix elements $e^{-d q_{n}}$ where $q_{n}=\sqrt{k^{2}+\kappa_{n}^{2}} \equiv q_{n}(k), k=|\mathbf{k}|$ and $\kappa_{n}=\xi_{n} / c$. For the undeformed slab, the operator $\mathbb{T}^{(2)}$ is diagonal in the plane-wave basis with matrix elements given by the scattering amplitudes

$$
\mathcal{T}_{Q Q^{\prime}}^{(2)}\left(\mathbf{k}, \mathbf{k}^{\prime}\right)=(2 \pi)^{2} \delta^{(2)}\left(\mathbf{k}-\mathbf{k}^{\prime}\right) \delta_{Q Q^{\prime}} r_{Q}^{(2)}\left(i \xi_{n}, \mathbf{k}\right),
$$

where $r_{Q}^{(j)}\left(i \xi_{n}, \mathbf{k}\right)$ are the Fresnel reflection coefficients

$$
r_{E}^{(j)}\left(i \xi_{n}, k\right)=\frac{\epsilon_{j}\left(i \xi_{n}\right) q_{n}-s_{n}^{(j)}}{\epsilon_{j}\left(i \xi_{n}\right) q_{n}+s_{n}^{(j)}}, r_{M}^{(j)}\left(i \xi_{n}, k\right)=\frac{q_{n}-s_{n}^{(j)}}{q_{n}+s_{n}^{(j)}},
$$

and $s_{n}^{(j)}=\sqrt{\epsilon_{j}\left(i \xi_{n}\right) \kappa_{n}^{2}+k^{2}}$. There are no analytical formulae for the elements of the T-operator of the curved plate $\mathbb{T}^{(1)}$, but for small deformations they can be expanded in powers of $h(\mathbf{x})$ as ${ }^{15}$

$$
\begin{aligned}
& \mathcal{T}_{Q Q^{\prime}}^{(1)}\left(\mathbf{k}, \mathbf{k}^{\prime}\right)=(2 \pi)^{2} \delta^{(2)}\left(\mathbf{k}-\mathbf{k}^{\prime}\right) \delta_{Q Q^{\prime}} r_{Q}^{(1)}\left(i \xi_{n}, \mathbf{k}\right) \\
& +\sqrt{q_{n} q_{n}^{\prime}}\left[-2 B_{Q Q^{\prime}}\left(\mathbf{k}, \mathbf{k}^{\prime}\right) \tilde{h}\left(\mathbf{k}-\mathbf{k}^{\prime}\right)\right. \\
& \left.+\int \frac{d^{2} \mathbf{k}^{\prime \prime}}{(2 \pi)^{2}}\left(B_{2}\right)_{Q Q^{\prime}}\left(\mathbf{k}, \mathbf{k}^{\prime} ; \mathbf{k}^{\prime \prime}\right) \tilde{h}\left(\mathbf{k}-\mathbf{k}^{\prime \prime}\right) \tilde{h}\left(\mathbf{k}^{\prime \prime}-\mathbf{k}^{\prime}\right)+\ldots\right]
\end{aligned}
$$

where $q_{n}^{\prime}=q_{n}\left(k^{\prime}\right)$. The coefficients $B_{Q Q^{\prime}}\left(\mathbf{k}, \mathbf{k}^{\prime}\right)$ and $\left(B_{2}\right)_{Q Q}\left(\mathbf{k}^{\prime}, \mathbf{k}^{\prime} ; \mathbf{k}^{\prime \prime}\right)$ can be obtained by standard perturbation theory in the height field and are given in Ref. 15; they depend on the relative orientation of the wave vectors $\mathbf{k}$ and $\mathbf{k}^{\prime}$, on the corresponding $q_{n}$ and $s_{n}$ and the dielectric function itself. Substituting the above expansion into Eq. (6), we obtain

$$
\tilde{G}(k ; d)=k_{B} T \sum_{n \geq 0}^{\prime} \int \frac{d^{2} \mathbf{k}^{\prime}}{(2 \pi)^{2}} \frac{f_{n}\left(\mathbf{k}^{\prime}, \mathbf{k}^{\prime}+\mathbf{k}\right)+f_{n}\left(\mathbf{k}^{\prime}, \mathbf{k}^{\prime}-\mathbf{k}\right)}{2},
$$

where

$$
\begin{aligned}
& f_{n}\left(\mathbf{k}^{\prime}, \mathbf{k}^{\prime \prime}\right)=-\sum_{Q} \frac{q_{n}^{\prime} r_{Q}^{(2)}\left(k^{\prime}\right)}{g_{Q}\left(k^{\prime}\right)} e^{-2 q_{n}^{\prime} d}\left[\left(B_{2}\right)_{Q Q}\left(\mathbf{k}^{\prime}, \mathbf{k}^{\prime} ; \mathbf{k}^{\prime \prime}\right)\right. \\
& \left.+2 \sum_{Q^{\prime}} \frac{q_{n}^{\prime \prime} r_{Q^{\prime}}^{(2)}\left(k^{\prime \prime}\right)}{g_{Q^{\prime}}\left(k^{\prime \prime}\right)} e^{-2 q_{n}^{\prime \prime} d} B_{Q Q^{\prime}}\left(\mathbf{k}^{\prime}, \mathbf{k}^{\prime \prime}\right) B_{Q^{\prime} Q}\left(\mathbf{k}^{\prime \prime}, \mathbf{k}^{\prime}\right)\right]
\end{aligned}
$$

with $q_{n}^{\prime \prime}=q_{n}\left(k^{\prime \prime}\right), g_{Q}(k)=1-r_{Q}^{(1)} r_{Q}^{(2)} \exp \left[-2 q_{n} d\right]$. The explicit dependence of several quantities on $i \xi_{n}$ is not shown for brevity. Since the sum over Matsubara frequencies in Eq. (10) is exponentially convergent, the existence of the second $k$-derivative of $\tilde{G}(k ; d)$ is ensured if for all $n \geq 0$ the second derivative of $f_{n}\left(\mathbf{k}^{\prime}, \mathbf{k}^{\prime}+\mathbf{k}\right)$ with respect to say $k_{x}$, is absolutely integrable over $\mathbf{k}^{\prime}$ for $\mathbf{k}=\mathbf{0}$. We then have

$$
\alpha(d)=\left.\frac{1}{2} \frac{\partial^{2} \tilde{G}}{\partial k^{2}}\right|_{k=0}=\left.\frac{k_{B} T}{2} \sum_{n \geq 0}^{\prime} \int \frac{d^{2} \mathbf{k}^{\prime}}{(2 \pi)^{2}} \frac{\partial^{2} f_{n}\left(\mathbf{k}^{\prime}, \mathbf{k}^{\prime}+\mathbf{k}\right)}{\partial k_{x}^{2}}\right|_{\mathbf{k}=\mathbf{0}}
$$

Having obtained the amplitude function $\alpha(H)$ by a comparison with the perturbative free energy at small in-plane momenta, we note that in principle, the free energy of Eq. (1) should follow directly from Eq. (6) when the T-operators are expanded in powers of the gradient of the surface profile $h(\mathbf{x})$, making no assumption about the amplitude of $h(\mathbf{x})$ itself. Indeed, such an expansion has been carried out for dielectric surfaces in Ref. 15. Substitution of this gradient expansion in Eq. (6) yields a nonlocal functional for the free energy. Hence, the function $\alpha(H)$ must be determined by the gradient expansion of the T-operator and subsequent locality expansion of the free energy. ${ }^{16}$

The above formulae enable evaluation of $\alpha(d)$ for arbitrary dielectric functions $\epsilon_{1}(\omega)$ and $\epsilon_{2}(\omega)$. In the ideal limit of perfect conductors at zero temperature (as well as for scalar fields obeying Dirichlet, Neumann and mixed boundary conditions $), \alpha(d)$ has a simple power law dependence on $d$; as $\alpha(d) \sim 1 / d^{3}$ with a coefficient that can be computed exactly. This permits to obtain simple closed formulae for the leading correction to the PFA for a variety of profiles $h(\mathbf{x}) \cdot{ }^{11}$ In general, for finite $T$ and/or for dielectric materials, the dependence of $\alpha(d)$ on $d$ cannot be expressed as a simple power law, and has 
to be computed numerically. Evaluation of Eq. (1) then permits to estimate the leading correction to PFA for arbitrary shapes of the profiles, and for any materials and temperatures of the involved bodies.

While numerical evaluation of Eq. (1) for specific experimental setups (e.g. spheres, cylinders or corrugated plates) is straightforward in general, by simple manipulations of Eq. (1) not presented here for brevity ${ }^{16}$, it is possible to obtain a closed semi-analytical expression for the leading correction to PFA for the gradient of the Casimir force $\partial F / \partial d=-\partial^{2} \mathcal{F} / \partial d^{2}$ (a quantity that is measured directly in some experiments ${ }^{17}$ ), in the experimentally relevant geometry of an arbitrary but axially symmetric profile $h(\mathbf{x})$, which is a smooth function of $\rho^{2}=x_{1}^{2}+x_{2}^{2}$. We expand the profile around the point of minimum separation as

$$
h\left(x_{1}, x_{2}\right) \equiv h\left(\rho^{2}\right)=d+\frac{\rho^{2}}{2 R}+c_{1} \frac{\rho^{4}}{2 R^{3}}+\ldots,
$$

where $R$ sets the overall scale of curvature. By substituting the above expansion into Eq. (1), one can derive the leading correction to PFA for the force-gradient as

$$
\frac{\partial F}{\partial d}=-2 \pi R F_{p p}(d)\left(1+\hat{\theta}_{1} \frac{d}{R}+o(d / R)\right),
$$

where $F_{p p}(d)=-\partial \mathcal{F}_{p p}(d) / \partial d$ is the Casimir force per unit area between two parallel plates, and

$$
\hat{\theta}_{1}=\frac{\mathcal{F}_{p p}(d)}{d F_{p p}(d)}\left(2 \beta(d)-4 c_{1}\right), \quad \beta(d)=\frac{\delta(d)}{\mathcal{F}_{p p}(d)} .
$$

Note that the dimensionless function $\beta(d)$ depends on the ratio of $d$ to one (or more) material dependent length scale. Independently of the dielectric material, the function $\delta(d)$ becomes a simple power law in the non-retarded limit where $\mathcal{F}_{p p}(d) \sim 1 / d^{2}$ and $\delta(d) \sim 1 / d^{2}$ so that $\beta(d)$ and hence $\hat{\theta}_{1}$ tends to a constant for $d \rightarrow 0$. This implies that in the non-retarded limit the first correction to the PFA for $\mathcal{F}$ scales logarithmically with the separation $d$.

Using the above formulae, we numerically computed the coefficient $\hat{\theta}_{1}$ in the experimentally relevant sphereplate geometry (corresponding to $c_{1}=1 / 4$ ), assuming for simplicity that both surfaces are made of the same material; gold at $T=300 \mathrm{~K}$. For a perfect reflector at zero temperature, an exact value for $\hat{\theta}_{1}$ can be obtained. In this limit the coefficient $\beta$ was computed in Ref. 11, and given as $\beta_{\mathrm{EM}}=2 / 3\left(1-15 / \pi^{2}\right)$. Since for perfect reflectors at $T=0, \mathcal{F}_{p p}=-\pi^{2} \hbar c /\left(720 d^{3}\right)$, it follows from Eq. (15) that $\left.\hat{\theta}_{1}\right|_{T=0} ^{\epsilon=\infty}=\frac{1}{3}\left(2 \beta_{\mathrm{EM}}-1\right)=-0.564$. Away from this ideal limit, $\hat{\theta}_{1}$ has to be computed numerically. In our computations for gold, we adopted the Drude model (for a discussion of alternative models see Ref. 18, Chap. 13). In this model, the permittivity $\epsilon(i \xi)$ to be used in the computation of the Casimir force for (ohmic) conductors is obtained using the well known Kramers-Kronig relation. For $\operatorname{Im}[\epsilon(\omega)]$ we used the tabulated optical data for gold quoted in Ref. 19, extrapolated to zero frequency by means of the Drude model, with parameters $\Omega_{p}=9 \mathrm{eV} / \hbar$ and $\gamma=35 \mathrm{meV} / \hbar$ (see Ref. 18, p.336). The resulting $\hat{\theta}_{1}$ is plotted in Fig. 2, as a function of $\log _{10}\left(2 \pi d / \lambda_{300 \mathrm{~K}}\right)$, where $\lambda_{T}=\hbar c /\left(k_{B} T\right)$ is the thermal wavelength $\left(\lambda_{300 \mathrm{~K}}=7.6 \mu \mathrm{m}\right)$. The inset of Fig. 2 shows these data versus the separation in linear scale (in microns). The crosses in Fig. 2 correspond to data for $T=300 \mathrm{~K}$, while the dashed lines are for $T=0 \mathrm{~K}$. We also show (solid lines) the result for perfect reflectors at $T=300 \mathrm{~K}$. Thus, the solid lines neglect finite conductivity corrections, while the dashed lines neglect finite temperature corrections. The importance of including both corrections for the leading (PFA) Casimir force has been previously noted ${ }^{20}$. A comparable previous estimate of $\hat{\theta}_{1}$, for a metal sphere-plate setup at zero temperature, was obtained in Ref. 21. This estimate is based on a numerical summation of a partial wave series, valid at large separations $d \gg R$, and fitting the results at short separations $d \rightarrow 0$ to a polynomial series. While our explicit computation here confirms a linear correction to PFA as in Eq. (14), we expect higher order corrections to involve non-polynominal logarithmic terms ${ }^{11}$.

Some comments about the qualitative behavior of the numerical results are in order. As expected, the $T=0$ perfect conductor limit of -0.564 is approached by the perfect conductor data at $T=300 \mathrm{~K}$ (solid lines) for short separations, which is where temperature effects become negligible; while the $T=0$ data for gold (dashed lines) approach this limit for large separations, which is where the skin depth becomes negligible. We observe also that in the limit of large separations, the $T=300 \mathrm{~K}$ data in Fig. 2 (crosses and the solid line), both approach the limit $\hat{\theta}_{1}(d \rightarrow \infty)=1 /(12 \zeta(3))=0.0693$, which coincides with the classical high-temperature limit of a scalar field obeying Dirichlet boundary conditions. This is plausible since for finite $T$ in the limit of large separations the Casimir force is dominated by classical thermal fluctuations of the electromagnetic field. It is well known that for non-magnetic materials like ohmic conductors, transverse electromagnetic fields decouple in the classical limit (Bohr-van Leeuwen theorem ${ }^{22}$ ), and the Casimir force arises entirely from low-frequency fluctuations of the longitudinal electric field (non-retarded limit of E modes), associated with scalar potentials which vanish on the surface of a conductor.

Importantly, we see from Fig. 2 that the data for gold at $T=300 \mathrm{~K}$ deviate significantly from both the solid and the dashed curves for separations $d$ between $150 \mathrm{~nm}$ and $300 \mathrm{~nm}$, showing that in this range a precise determination of $\hat{\theta}_{1}$ requires simultaneous consideration of both finite conductivity and finite $T$ corrections. This is somewhat surprising since the thermal correction to the Casimir force between two metallic plates is small for such small separations (in the Drude model, less than 3\% below $200 \mathrm{~nm}$ ). On the contrary, we see from Fig. 2 that the magnitude of the thermal correction to $\hat{\theta}_{1}$ is about $20 \%$ for $d=200 \mathrm{~nm}$, indicating that $\hat{\theta}_{1}$ is much more sensitive to temperature than the Casimir force itself. For a 


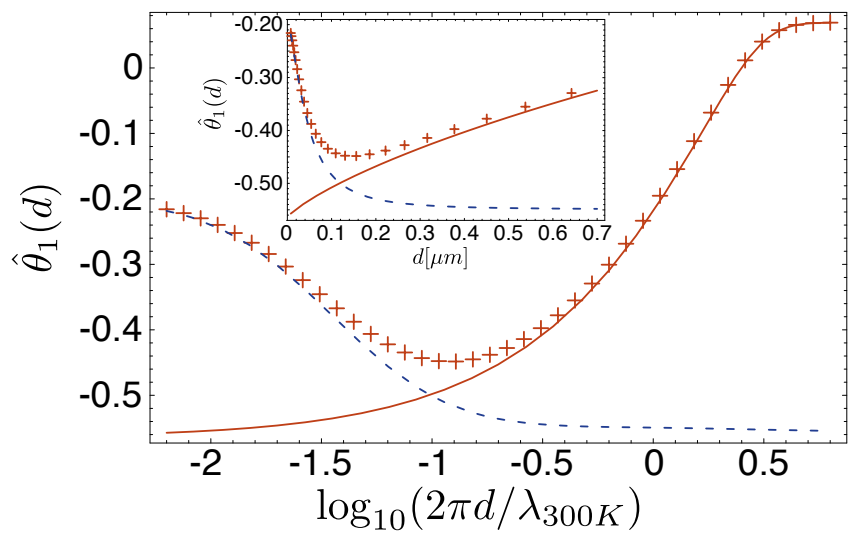

FIG. 2. $\hat{\theta}_{1}$ for a gold sphere in front of a gold plate, computed using the tabulated optical data for gold, see Ref. 19. Crosses correspond to $T=300 \mathrm{~K}$, while the dashed line is for $T=0 \mathrm{~K}$. The solid line is for an ideal conductor at $T=300 \mathrm{~K}$. For ideal conductors at $T=0, \hat{\theta}_{1}=-0.564$ independently of separation. The inset depicts the same data, for gold at $300 \mathrm{~K}$, as a function of separation (in microns) on a linear scale.

vanishing separation we get $\hat{\theta}_{1}(d \rightarrow 0)=-0.206$ which is consistent with our general prediction of a finite $\hat{\theta}_{1}$ in the non-retarded limit, and suggests that $\hat{\theta}_{1}(d)$ is a bounded function.

Since Casimir force measurements are often limited to rather short separations between weakly curved surfaces, our work has immediate experimental relevance. An attempt to measure the coefficient $\hat{\theta}_{1}$ for a gold plate-sphere geometry at room temperature was made by using a micromachined torsional oscillator. ${ }^{23}$ The best estimate of $\hat{\theta}_{1}$ was obtained by dynamically measuring the Casimir force gradient at fixed separation $d$, for five spheres of different radii $R$, ranging from 10.5 to $148.2 \mu \mathrm{m}$, and fitting the data linearly in $1 / R$. It was estimated that $\hat{\theta}_{1}$ is less than 0.4 at $95 \%$ confidence level, in the range $164 \mathrm{~nm}$ $<d<300 \mathrm{~nm}$. Our data in Fig. 2 are slightly above, but roughly consistent with this bound. The small disagreement between our estimate of $\hat{\theta}_{1}$ and the experimental bound may be explained by observing that the theoretical prediction of $\hat{\theta}_{1}$ is sensitive to the plasma frequency $\omega_{p}$, with smaller $\omega_{p}$ leading to smaller values for $\hat{\theta}_{1}$. In our computations we used the standard value for gold $\left(\omega_{p}=9 \mathrm{eV} / \hbar\right)$, but it is well known that gold films produced by deposition techniques may have much smaller values of the plasma frequency. ${ }^{24}$ Another consideration is that, when fitting the force-gradient data in Ref. 23 higher order corrections, of magnitude $\hat{\theta}_{2}(d / R)^{2}$, to PFA were neglected. The coefficient $\hat{\theta}_{2}$ cannot be computed analytically yet, but its magnitude was estimated for perfect conductors at zero temperature through a Padé extrapolation constrained by a multipole expansion at large separations and by the gradient expansion at short separation. ${ }^{11}$ It appears that $\hat{\theta}_{2}$ is positive and of order one. Neglect of this second order correction in the fits of Ref. 23, especially for the smallest sphere, may lead to a systematic underestimation of $\hat{\theta}_{1}$. Finally, it would be important to investigate the influence of surface roughness on $\hat{\theta}_{1}$.

This research was supported by the ESF Research Network CASIMIR (GB, TE), and NSF Grant No. DMR-08$03315(\mathrm{MK})$.

${ }^{1}$ H. B. G. Casimir, Proc. K. Ned. Akad. Wet. 51, 793 (1948).

${ }^{2}$ V. A. Parsegian, Van der Waals Forces (Cambridge University Press, Cambridge, England, 2005).

${ }^{3}$ M. Rose, Photonics Spectra 42, 77 (2008).

${ }^{4}$ M. T. H. Reid, A. W. Rodriguez, J. White, and S. G. Johnson, Phys. Rev. Lett. 103, 040401 (2009).

${ }^{5}$ H. Gies and K. Klingmüller, Phys. Rev. D 74, 045002 (2006).

${ }^{6}$ E. M. Lifshitz, Sov. Phys. JETP 2, 73 (1956).

${ }^{7}$ B. Derjaguin, Kolloid Z. 69, 155 (1934).

${ }^{8}$ A. Rodriguez, M. Ibanescu, D. Iannuzzi, F. Capasso, J. D. Joannopoulos and S. G. Johnson, Phys. Rev. Lett. 99, 80401 (2007).

${ }^{9}$ The general case of two curved surfaces can always be reduced to this one, by exploiting the tilt invariance of the Casimir energy. ${ }^{11}$

${ }^{10}$ C. D. Fosco, F. C. Lombardo, and F. D. Mazzitelli, Phys. Rev. D 84, 105031 (2011).

${ }^{11}$ G. Bimonte, T. Emig, R. L. Jaffe, and M. Kardar, e-print arXiv:1110.1082, to appear in Europhys. Lett. (2012).

${ }^{12}$ To be precise, consider the one-parameter family of profiles $\left.\left.H_{\lambda}(\mathbf{x})=H(\lambda \mathbf{x}), \lambda \in\right] 0,1\right]$ possessing finite derivatives up to second order. For small $\lambda$ the leading term $\mathcal{F}_{\mathrm{PFA}}[H]$ in Eq. (1) is generically of order $1 / \lambda^{2}$ (for bi-directionally curved surfaces), while the first correction proportional to $(\nabla H)^{2}$ is of order $\lambda^{0}$. Then, for the gradient expansion in Eq. (1) to be valid, we must have $\rho^{(2)}\left[H_{\lambda}\right]=o\left(\lambda^{0}\right)$, where $o\left(x^{n}\right)$ denotes an infinitesimal of order higher than $n$, such that $\lim _{x \rightarrow 0} o\left(x^{n}\right) / x^{n}=0$.

${ }^{13}$ S. J. Rahi, T. Emig, N. Graham, R. L. Jaffe, and M. Kardar, Phys. Rev. D 80, 085021 (2009).

${ }^{14}$ We normalize the waves $|\mathbf{k}, Q\rangle$ as in Ref. 15 . Note though that the choice of normalization is irrelevant for the purpose of evaluating the trace in Eq. (6).

${ }^{15}$ A. Voronovich, Waves Rand. Media, 4, 337 (1994).

${ }^{16}$ G. Bimonte, T. Emig, R. L. Jaffe and M. Kardar, unpublished.

${ }^{17}$ R.S. Decca, D. Lopez, E. Fischbach, G.L. Klimchitskaya, D.E. Krause, and V.M. Mostepanenko, Eur. Phys. J. C 51, 963 (2007).

${ }^{18}$ Advances in the Casimir Effect, M. Bordag, G. L. Klimchitskaya, U. Mohideen, V. M. Mostepanenko, (Oxford University Press, 2009).

${ }^{19}$ Handbook of Optical Constants of Solids, E. D. Palik, (Academic, New York, 1995).

${ }^{20}$ A. C. Canaguier, P. A. M. Neto, A. Lambrecht, S. Renaud, Phys. Rev. Lett. 104, 040403 (2010).

${ }^{21}$ A. C. Canaguier, P. A. M. Neto, I. Cavero-Pelaez, A. Lambrecht, S. Reynaud, Phys. Rev. Lett. 102, 230404 (2009).

${ }^{22}$ G. Bimonte, Phys. Rev. A 79, 042107 (2009).

${ }^{23}$ D. E. Krause, R. S. Decca, D. Lopez, and E. Fischbach, Phys. Rev. Lett. 98, 050403 (2007).

${ }^{24}$ V. B. Svetovoy, P. J. van Zwol, G. Palasantzas, and J. Th. M. De Hosson, Phys. Rev. B 77, 035439 (2008). 